\title{
PATENTOMETRIA: UMA FERRAMENTA INDISPENSÁVEL NO ESTUDO DE DESENVOLVIMENTO DE TECNOLOGIAS PARA A INDÚSTRIA QUÍMICA
}

\author{
Marcelo Gomes Speziali ${ }^{\mathrm{a}, *,(\odot) ~}$ e Raphael da Silva Nascimento ${ }^{\mathrm{b}}$ \\ aDepartamento de Química, Instituto de Ciências Exatas e Biológicas, Universidade Federal de Ouro Preto, Campus Universitário \\ Morro do Cruzeiro, 35400-000 Ouro Preto - MG, Brasil

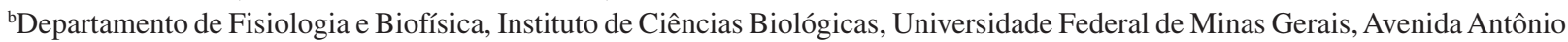 \\ Carlos 6627, Pampulha, 31270-901 Belo Horizonte - MG, Brasil
}

Recebido em 26/05/2020; aceito em 03/07/2020; publicado na web em 27/08/2020

\begin{abstract}
PATENTOMETRY: AN INDISPENSABLE TOOL IN THE STUDY OF THE DEVELOPMENT OF TECHNOLOGIES FOR THE CHEMICAL INDUSTRY. This work describes a methodology to carry out the analysis of technological sectors, using information contained in patent documents. The use of this information by professors and students of chemistry courses and related areas is still little used. The consequence of this is the repetition of efforts to obtain some results of research and development. The use of patent big data and the construction of scenarios, using several variables, is even rarer in Brazil and still little explored in the world. This work presents patentometry in a theoretical context and its practical application, taking as an example the area of rare earth in Brazil. Analyses will be presented in this work, such as the development of technologies over time and study of the maturity of the sector in question; potential markets; the most significant technology developers in the field; technological sectors related to rare earth in Brazil; attractiveness of the Brazilian market; the evolution of priority sectors; technological specialization; patent quality indices and innovation indices; indexes of technologies internationalization and cooperation. It is expected that with the Patentometry, some of the barriers of insertion in the market of new technologies will be better known and minimized.
\end{abstract}

Keywords: big data; patents; innovation; data mining; indicators; rare earth.

\section{INTRODUÇÃO}

Dentre as várias formas de se acompanhar o desenvolvimento de uma tecnologia, um setor tecnológico ou de um grupo de empresas, certamente a prospecção tecnológica é uma das aliadas de maior importância nesse processo.

As ferramentas de prospecção tecnológica, mapeamentos, análise de big data e até mesmo o uso estratégico das informações tecnológicas contidas em documentos de patentes são subutilizados no Brasil. A combinação desses tipos de análise na patentometria é quase uma novidade absoluta entre os pesquisadores nas universidades e centros industriais de P\&D brasileiros. ${ }^{1}$ Buscas no estado da técnica e da arte, utilizando depósitos de patente podem nos dar uma visão mais assertiva sobre: os desdobramentos nas pesquisas com focos tecnológicos; possibilidades de se depositar novas patentes; inspiração na elaboração de projetos e até mesmo a apropriação legal de tecnologias não protegidas em um determinado território durante uma determinada época. A análise patentométrica pode ser uma valiosa ferramenta para auxiliar tomadores de decisões sobre a maturidade e a performance tecnológica, tecnologias emergentes, vieses e dinâmica de evolução de setores tecnológicos, a geografia do desenvolvimento da tecnologia e mercados de interesse, redes de colaboração, componentes de valoração de tecnologias em desenvolvimento, principais atores envolvidos, globalização da P\&D\&I, estratégias de proteção de empresas, previsões de surgimento de novas tecnologias, ciclos de vida e velocidade de difusão de uma tecnologia, necessidade de se criar redes de colaboração, dentre outros..$^{2-4}$

A patentometria certamente pode ser uma valiosa ferramenta para os profissionais da química, tanto acadêmicos quanto para os diretamente envolvidos no setor produtivo na hora de se tomar decisões relativas ao direcionamento do P\&D\&I de sua instituição. ${ }^{5}$

*e-mail: speziali@ufop.edu.br
A Organização Mundial para a Propriedade Intelectual (OMPI) publicou em 2015 uma série de boas práticas para o preparo de relatórios de mapeamentos de patentes. ${ }^{6}$ Além disso, no site https://www. wipo.int/patentscope/en/programs/patent_landscapes/ da própria OMPI ainda pode ser encontrada uma coletânea de diversos tipos de mapeamentos tecnológicos das mais diversas áreas e com consulta gratuita. É bom lembrar que, por fazer parte da inteligência estratégica de algumas empresas, relatórios de mapeamentos tecnológicos mais completos não são encontrados de forma gratuita.

Estudos de prospecção, que utilizam métodos quantitativos ou qualitativos, são importantes para facilitar a conceitualização de soluções plausíveis para problemas no estado da técnica e da arte. Dentre os mais diversos modos de se analisar tecnologias, utilizando a patentometria, Kupfer e Tigre, ${ }^{7}$ assim como Teixeira ${ }^{8}$ classificaram métodos de análise e de prospecção de tecnologias em três diferentes segmentos: monitoramento; métodos de previsão baseados em Forecasting e Foresight. Um resumo dos principais modos de se fazer uma prospecção tecnológica é apresentado no trabalho de Coelho (2003, apud Teixeira, 2013); 9 são eles: análise da inteligência competitiva tecnológica; análise de tendências; opiniões de experts; construção de cenários; métodos computacionais/ferramentas analíticas.

A importância de utilizar documentos de patentes como fonte de informação tecnológica foi salientada em publicações da Organização para a Cooperação e Desenvolvimento Econômico (OCED) ${ }^{10}$ e da revista Cadernos de Prospecção Tecnológica publicado pela editora da UFBA e com o apoio do mestrado profissional PROFNIT e do Fórum Nacional de Gestores de Inovação e Transferência de Tecnologia (FORTEC). Nesse sentido, o grupo de pesquisa ao qual os autores fazem parte vem desenvolvendo técnicas de patentometria para os mais diversos setores tecnológicos, dentre eles, hidrometalurgia, líquidos iônicos e flavorizantes e fragrâncias. ${ }^{11-13}$

O processo de análise de big data de patentes pode ser feito de diversas formas e com diversas finalidades. Abbas et al., em seu 
trabalho publicado na World Patent Information, ${ }^{14}$ exemplificou em um fluxograma a trajetória da busca e processamento de documentos de patentes, aplicando os resultados em algumas áreas estratégicas, como pode ser visto de forma adaptada na Figura 1.

Conforme Figura 1, no bloco i) são citadas diversas bases de dados de patentes, tanto gratuitas quanto comerciais. Vale lembrar que todas as bases de dados possuem suas limitações, como abrangência territorial e frequência na atualização das informações. Para se trabalhar com patentometria é interessante que se utilize, pelo menos, mais de uma base de dados, de forma a minimizar esses erros intrínsecos das próprias bases. ${ }^{12}$ Para a exportação dos dados no bloco ii), algumas bases de dados já oferecem o suporte para arquivos nos formatos $t x t, c s v, x l s$, dentre outros. Dependendo do software que o analista pretenda trabalhar na estruturação e consolidação dos dados - blocos iii) e iv) - um ou outro formato será preterido. É importante lembrar que as duplicidades surgem na recuperação de grandes volumes de dados, não apenas pela sobreposição de bases de dados distintas, mas também por questões oriundas nos próprios escritórios regionais de patentes, que algumas vezes publicam repetidas vezes o mesmo pedido, pelas mais diversas razões. Caso o analista não execute corretamente a sugestão do bloco v), certamente os resultados da análise será enviesado, uma vez que apontará para falsas direções obtidas pela repetição desnecessária de documentos. A construção dos mapas de visualização e da matriz de análise no bloco vi) está intimamente relacionada com a especialização técnica do analista executor do procedimento, uma vez que pessoas com diferentes formações podem realizar interpretações distintas sobre as informações geradas nesta etapa.

A patentometria é uma área altamente transdisciplinar, sendo assim, é interessante que profissionais das mais diversas áreas trabalhem juntos para obter a interpretação correta e ampla dos cenários obtidos pela análise de grandes volumes de dados de patentes.

A área de Cheminformatics utiliza o Machine Learning e Inteligência Artificial, de forma altamente transdisciplinar, para auxiliar na busca pela descoberta de novas drogas, ${ }^{15}$ prever reações químicas ${ }^{16,17}$ e estruturas moleculares a partir de dados brutos de RMN. ${ }^{18}$ No entanto, poderia utilizar a patentometria para prever o desenvolvimento e tendências de lançamentos futuros de produtos químicos, além de identificar setores tecnológicos promissores ou possíveis de gerar inovações disruptivas.

Neste contexto, o presente artigo irá abordar de forma simplificada os primeiros passos para se construir um panorama tecnológico utilizando a patentometria. Pretende-se informar o leitor sobre as possibilidades de: construir cenários utilizando grandes volumes de dados obtidos em documentos de patentes; relacionar esses dados com o desenvolvimento da química; utilizar o resultado da análise no auxílio da P\&D\&I realizadas pelos profissionais da química. Serão abordados de forma simplificada como se utilizar indicadores de desenvolvimento e qualidade de tecnologias, utilizando ferramentas da econometria aplicadas a química. Após a explicação teórica das ferramentas mais utilizadas pela patentometria, serão apresentados ao leitor os resultados de um mapeamento hipotético sem a interpretação específica do setor de análise. No caso exemplificado pelo presente artigo, utilizaremos as patentes depositadas no Brasil com o assunto de Terras Raras. Assim, espera-se que o leitor tenha contato com as ferramentas de análise e suas possibilidades. Ressalta-se que a patentometria é uma ferramenta fundamental para a construção de cenários e mapeamentos de desenvolvimento tecnológico, porém a interpretação desses mapas e cenários dependerá do background técnico do analista. Dependendo do conhecimento prévio do analista a interpretação desses dados poderá tomar um foco mais técnico, mais econômico, mais legalista, uma vez que a patentometria é uma ferramenta de utilidade transdisciplinar.

\section{Metodologia de construção do panorama de tecnologias}

Para a construção de mapas de desenvolvimento de um setor tecnológico, a patentometria utiliza diversas informações acerca do setor tecnológico em estudo, sendo que algumas dessas informações serão encontradas nos próprios documentos de patentes, mais especificamente nos campos definidos pelos códigos INID. ${ }^{19}$ Os principais códigos INID utilizados numa prospecção são:

(11) Número do documento $-X_{i}$;

(21) Número designado ao documento quando de seu depósito $-X_{2}$; (31) Número designado ao primeiro depósito (prioridade do documento) $-X_{3}$;

(22) Data de depósito da solicitação $-X_{4}$;

(32) Data de depósito da primeira solicitação (data de prioridade) $-X_{5}$;

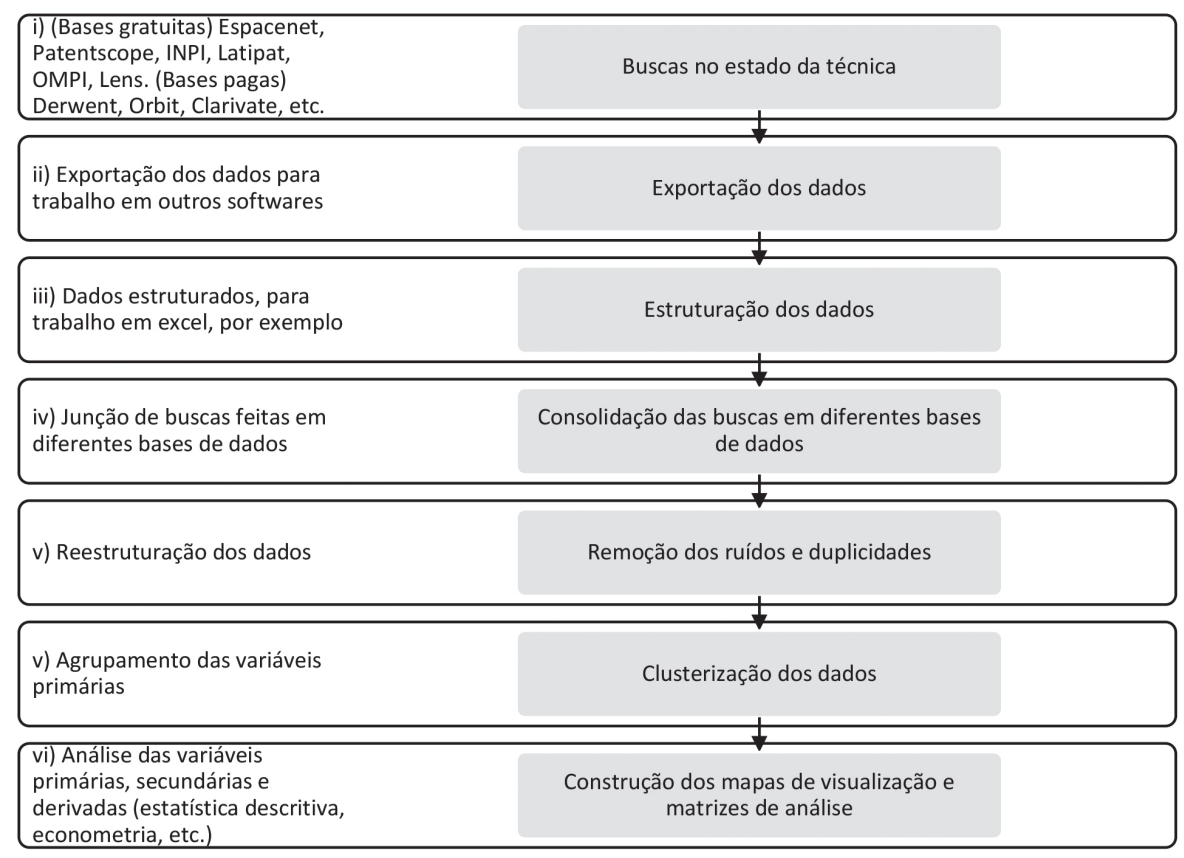

Figura 1. Fluxograma genérico de análise de patentes adaptado e baseado no trabalho de Abbas et al. ${ }^{14}$ 
(41) a (47) Referem-se a documentos de patentes publicados [examinados ou não, mas que ainda não têm a decisão final (concessão ou não da patente) $]-X_{6}$;

(45) a (47) Referem-se à data de concessão da patente, geralmente é usado o número (45) $-X_{7}$;

(19) Nome do país ou organização regional ou internacional que publicou o documento de patente $-X_{\dot{8}}$;

(33) País ou países do primeiro documento (prioridade) $-X_{9}$;

(70) a (76) Identificação de partes relacionadas com o documento $-X_{10}$;

(71) Nome do depositante $-X_{11}$;

(72) Nome do inventor, se conhecido $-X_{12}$;

(73) Nome de quem detém os direitos sobre a patente $-X_{13}$;

(74) Nome do procurador ou agente $-X_{14}$;

(75) Nome do inventor, quando também for o depositante $-X_{15}$;

(12) Tipo de documento (Patente de invenção, Modelo de utilidade,

etc.) $-X_{16}$;

(51) Classificação internacional de patente $-X_{17}$;

(52) Classificação nacional ou doméstica de patente $-X_{18}$;

(54) Título da invenção $-X_{19}$;

(56) Lista de documentos anteriores citados pelo depositante (pode auxiliar no exame) ou encontrados pelo examinador de patentes durante a busca para exame $-X_{20}$;

(57) Resumo do conteúdo do documento $-X_{21}$.

As variáveis $X_{n}$, após a definição dos códigos INID, são para propósitos matemáticos desse trabalho.

\section{Indicadores primários, derivados dos códigos INID}

Os códigos INID indicam de forma direta alguns fatos e tendências, quando analisados sob a ótica da patentometria. Por se encontrarem de forma explícita no documento de patente, chamamos os dados obtidos a partir da análise direta desses INID de indicadores primários. A exemplo do tipo de informação que pode ser obtida desses indicadores primários temos:

- Avaliação de depósitos de famílias de patentes pelo mundo ao longo dos anos. $\mathrm{O}$ ano do primeiro depósito (earliest filing date) é o mais indicado para ser utilizado, pois se refere à data mais próxima à concepção da tecnologia. Essa análise também é conhecida como série temporal ou simplesmente $S T$ :

$$
S T=\sum f\left(X_{5(t)}\right) \text { Vs tempo }_{(t)}
$$

- Avaliação dos potenciais mercados de proteção, feita pela análise dos países onde foram feitos os depósitos totais de patentes (incluindo as patentes filhas, ou seja, todas as patentes pertencentes à mesma família). Essa informação é obtida nos campos de famílias de patente ou INPADOC. O principal objetivo de uma patente é impedir que um concorrente explore aquela tecnologia sem a autorização prévia do seu titular. Dessa forma, um depositante patenteia sua tecnologia em determinadas localidades, em que se julgue serem mercados onde a concorrência precise ser inibida. Esses locais são chamados de mercados de proteção ou $M P$ :

$$
M P=\sum f\left(\operatorname{cod}_{1}\right) ; \sum f\left(\operatorname{cod}_{2}\right) ; \sum f\left(\operatorname{cod}_{3}\right) ; \sum f\left(\operatorname{cod}_{n}\right)
$$

sendo o $\operatorname{cod}_{(n)}$ formado pelas duas letras iniciais de $X_{1}$. Exemplos: $X_{1}=\mathrm{RU} 2595178 \mathrm{C} 2, \sum f\left(\operatorname{cod}_{1}\right)$ seria a somatória de todas as patentes com o código RU; $X_{1}=\mathrm{CN} 101705380 \mathrm{~B}, \Sigma f\left(\operatorname{cod}_{2}\right)$ seria a somatória de todas as patentes com o código $\mathrm{CN}$.

- Avaliação dos depositantes das patentes $(D P)$. Atenção especial deve ser dada nesse ponto, pois há depositantes que possuem mais de uma forma de designação, a exemplo: BASF Corporation, BASF SE, BASF GMBH, Givaudan AG, Givaudan Nederland Services, Petrobrás, Petróleo Brasileiro S.A., assim como universidades: UFOP, Universidade Federal de Ouro Preto, Univ. Fed. de Ouro Preto:

$$
D P=\sum f\left(X_{11(1)}\right) ; \sum f\left(X_{11(2)}\right) ; \sum f\left(X_{11(3)}\right) ; \sum f\left(X_{11(n)}\right)
$$

sendo $X_{11(\mathrm{n})}$ o nome de cada um dos depositantes, após agrupamentos por unicidades de nomes. Exemplos: $\sum f\left(X_{11(1)}\right)$ seria a somatória dos depositantes 4D Pharma PLC e 4D Pharma Res LTD como um único grupo de empresas. $\sum f\left(X_{11(2)}\right)$ seria a somatória de Abbott Cardiovascular Systems e Abbot Cardiovascular Systems Inc. que são um mesmo grupo de empresas. $\Sigma f\left(X_{11(3)}\right)$ seria Acad Sinica e Academia Sinica como um único grupo de depositantes.

- Avaliação das áreas técnicas $(A T)$ os quais os depósitos estão associados. Os códigos IPC e CPC são os principais instrumentos de qualificação dessa análise:

$$
A T=\sum f\left(X_{17(1)}\right) ; \sum f\left(X_{17(1)}\right) ; \sum f\left(X_{17(1)}\right) ; \sum f\left(X_{17(1)}\right)
$$

Nesse caso, $X_{17(\mathrm{n})}$ são os IPC, classes ou subclasses de IPC, conforme desejo do analista. Exemplos: $\Sigma f\left(X_{17(1)}\right)$ seria a somatória de todos os IPC A61K/70. $\Sigma\left(X_{11(2)}\right)$ seria a somatória de todos $\mathrm{C} 08 \mathrm{~J} 3 / 12$. $\sum\left(X_{11(3)}\right)$ seria a somatória de todos os IPC H.

- Avaliação dos centros produtores de tecnologias relacionadas à análise $(C P T)$. São utilizados os dados do escritório de onde ocorreu o depósito mais antigo (US, BR, JP, GB, FR, EP). Essa informação é obtida utilizando as duas primeiras letras de $X_{3}$, assim como na análise dos mercados de proteção. Essa análise está relacionada com o local de origem da tecnologia. Entretanto, é bom lembrar que, em alguns casos, o depositante poderá optar por fazer o depósito de prioridade em um país diferente do país de sua residência. Esses pequenos desvios da idealidade da análise entrarão no erro estatístico e serão minimizados com o uso de espaços amostrais maiores:

$$
C P T=\sum f\left(\operatorname{cod}_{1}\right) ; \sum f\left(\operatorname{cod}_{2}\right) ; \sum f\left(\operatorname{cod}_{3}\right) ; \sum f\left(\operatorname{cod}_{n}\right)
$$

- Análise das patentes triádicas ${ }^{20,21}$ (conforme definido pela OCDE, patentes triádicas são as patentes depositadas simultaneamente nos três maiores escritórios de patentes do mundo - JPO, EPO, USPTO). Devido ao alto investimento para se fazer depósitos simultaneamente nesses três escritórios, não é comum que um depositante os faça a menos que a tecnologia possua um real interesse. $\mathrm{O}$ indicador referente à análise das patentes triádicas é o PatT:

$$
P a t T=\sum f\left(X_{1(U S, E P, J P)}\right)
$$

- Análise da atratividade de mercado (Atr), utilizando a razão entre depósitos de residentes por depósitos de estrangeiros em um determinado país:

$$
\text { Atr }=\sum \frac{f\left(X_{11 \text { residentes }}\right)}{f\left(X_{11 \text { estrangeiros }}\right)}
$$

- Análise dos depositantes mais ativos (top10, top5, top3, topn) para a tecnologia ou setor em análise. O indicador para essa análise será o $D P n$, sendo (n) referente ao depositante analisado:

$$
D P_{n}=\sum f\left(X_{11(1)}\right) ; \sum f\left(X_{11(2)}\right) ; \sum f\left(X_{11(3)}\right) ; \sum f\left(X_{11(n)}\right)
$$


- Análise de nuvem de palavras $(N P)$, utilizando ferramentas de cloudwords nos campos do título, resumo ou reivindicações:

$$
N P=\sum f(F R P)
$$

sendo que FRP = frequência de repetição de palavras.

\section{Indicadores secundários, derivados dos códigos INID}

A relação entre códigos INID gerará indicadores derivados, que muitas das vezes serão responsáveis por resultados não intuitivos, porém importantes. Dentre as diversas formas de combinação, temos alguns exemplos:

- Análise da maturidade do campo tecnológico $(M T)$ pela construção da curva $S^{22}$ (quantidade cumulativa de depósitos de patentes ao longo dos anos):

$$
M T=\operatorname{ano}_{x} V s \sum f\left(d p_{a n o_{x}}\right) ; a n o_{x+1} V s \sum_{x}^{x+1} f\left(d p_{a o_{x}}\right) ; a n o_{x+n} V s \sum_{x}^{x=n} f\left(d p_{a n o_{x}}\right)
$$

sendo dp = número de depósitos.

- Série temporal para os IPC mais relevantes (evolução dos domínios tecnológicos em função do tempo - IPCT). Esse é um indicador derivado de AT em função do tempo:

$I P C T=\operatorname{tempo}_{(t)} V s \sum f\left(X_{17(t t)}\right) ;$ tempo $_{(t)} V_{S} \sum f\left(X_{17(2 t)}\right) ;$ tempo $_{(t)} V_{s} \sum f\left(X_{17(n t)}\right)$

sendo que $\sum f\left(X_{17 \mathrm{nt} t}\right)$ é a somatória da frequência de aparição do IPC analisado (n) para um determinado ano $(\mathrm{t})$.

- Série temporal de produção tecnológica dos principais depositantes (indicador da evolução histórica da produção tecnológica dos depositantes para uma determinada tecnologia ou setor em específico - $\left.D P_{n} T\right)$. Esse é um indicador derivado de $D P_{n}$ em função do tempo:

$$
D P_{n} T=\operatorname{tempo}_{(t)} V_{s} \sum f\left(X_{11(t i)}\right) ; \operatorname{tempo}_{(t)} V_{s} \sum f\left(X_{11(2 t)}\right) ; \operatorname{tempo}_{(t)} V_{s} \sum f\left(X_{11(n t)}\right)
$$

sendo que $\sum f\left(X_{11(\mathrm{nt})}\right)$ é a somatória da frequência de aparição do depositante analisado (n) para um determinado ano (t).

- Matriz de correlação de IPC com principais depositantes (indicador absoluto de especialização tecnológica dos depositantes - $E T_{n}$. Para o indicador $E T_{n}$, a variável (n) se refere ao depositante e (z) ao IPC analisado:

$$
E T_{n}=X_{11 n} V_{S} \sum f\left(X_{17 z}\right)
$$

ou

$$
E T_{n}=D P_{n} V s A T
$$

- Série temporal da produção tecnológica $(S P)$, na área de análise, para os depósitos em determinados países (evolução dos depósitos em determinadas regiões):

$$
S P=\sum f\left(\operatorname{cod}_{n}\right) V_{\text {sempo }_{(t)}}
$$

ou

$$
S P=S T V_{S} M P
$$

- Relação dos países em que as tecnologias são depositadas com a áreas técnicas (indicador de especialização tecnológica para determinadas regiões - ETP):

$$
E T P=\operatorname{cod}_{n} X \sum f\left(X_{17(z)}\right)
$$

Esses são apenas alguns exemplos da relação entre dois INID possíveis para gerar indicadores secundários. A combinação de uma ordem mais elevada de INID poderá gerar indicadores terciários ou de ordem superior, sendo aplicado de acordo com a experiência do analista.

\section{Indicadores ternários e de ordens mais elevadas}

Para a análise de indicadores ternários ou de ordens mais elevadas é necessário a construção de matrizes de relacionamento para visualizar os dados de forma correta. A título de ilustração, alguns desses indicadores podem ser assim definidos:

- Evolução temporal da especialização tecnológica de depositantes (relação entre a série temporal de IPC para determinados depositantes - STD):

$$
S T D=I P C T X X_{11}
$$

- Evolução temporal da especialização tecnológica de determinadas regiões (relação entre a série temporal de IPC para determinadas regiões - STP):

$$
S T P=I P C T X X_{1(\text { duas primeiras letras })}
$$

- Nuvens dinâmica de palavras, e outros $(N D)$ :

$$
N D=N P X \operatorname{tempo}(T)
$$

A relação de três ou mais variáveis obtidas dos códigos INID gerará vários tipos de indicadores de ordens superior conforme a necessidade do analista.

\section{Indicadores de patentes para monitoramento de competidores}

Alguns indicadores de inovação são mais sofisticados e, normalmente, derivam das análises da área econômica. Por outro lado, indicadores como especialização tecnológica e qualidade de patentes são menos complexos e muito utilizados para o monitoramento dos atores envolvidos na produção da tecnologia, ou do setor tecnológico em análise. A Tabela 1 traz alguns desses indicadores desenvolvidos pelo professor Holger Ernst. ${ }^{3}$

Outros índices de desenvolvimento tecnológico, são largamente discutidos na literatura ${ }^{2,23,24}$ a saber: Revealed Technology Advantage, Specialization Index, Revealed Patent Advantage Index, C2O Concentration Index, Generality of a patent, Foreign ownership of domestic inventions podem ser largamente utilizados com os dados obtidos pelos códigos INID. Não entraremos em detalhes sobre esses indicadores no artigo por se tratar de uma análise mais sofisticada e com a interpretação dos resultados quase sempre atreladas a um profissional da área da economia. Porém, para análises mais robustas sobre o desenvolvimento e uma possível aceitação pelo mercado de uma determinada tecnologia, é altamente recomendável que se faça um estudo setorial ou tecnológico mais completo, conforme finalidade determinada no início do processo.

\section{Exemplificação da construção de um panorama tecnológico sobre terras raras no Brasil}

De forma a ilustrar algumas das possibilidades de construção de um cenário das tecnologias sobre terras raras no Brasil, apresenta-se abaixo algumas das análises possíveis de serem feitas, utilizando informações de patentes. Para facilitar a compreensão da análise e minimizar o volume de dados a ser trabalhado, o big 
Tabela 1. Indicadores de patentes para monitoramento de competidores

\begin{tabular}{|c|c|c|c|}
\hline \multicolumn{4}{|c|}{ Indicador de Patente } \\
\hline Atividade patentária & $P A_{i F}=\sum P A_{T F i}$ & Escopo internacional & $Q_{3}=\sum P C T_{i F}$ \\
\hline Compartilhamento de tecnologias & $T S=\frac{P A_{i F}}{\sum_{i=1}^{i=\infty} P A_{i F}}$ & Frequência média de citações & $Q_{4}=\overline{\sum \text { citações }_{i F}}$ \\
\hline Ênfase em P\&D & $R \& D E=\frac{P A_{i F}}{\sum P A_{i}}$ & Qualidade média das patentes & $P Q_{i F}=\sum_{n=1}^{n=4} Q_{n}$ \\
\hline Intensidade de cooperação & $C I=P A_{\text {coop }_{i F}}$ & Força das patentes & $P S_{i F}=P Q_{i F} x P A_{i F}$ \\
\hline $\begin{array}{l}\text { Compartilhamento de tecnologias } \\
\text { concedidas }\end{array}$ & $Q_{1}=P A_{\text {conciF }}$ & Compartilhamento de tecnologias & $T S=\frac{P S_{i F}}{\sum_{i=1}^{i=\infty} P S_{i F}}$ \\
\hline Escopo tecnológico & $Q_{2}=\sum I C P_{i F}$ & $\begin{array}{l}\text { Compartilhamento relativo de tecno- } \\
\text { logias }\end{array}$ & $R T S=\frac{P S_{i F}}{M a ́ x P S_{i F}}$ \\
\hline
\end{tabular}

$\mathrm{i}$ ou $\mathrm{iF}=$ determinada empresa ou empresas de um determinado ramo tecnológico; $\mathrm{PA}=$ todas as patentes; $\mathrm{PA}_{\text {coop }}=$ patentes com Co titularidade; $\mathrm{PA}$ conc $=$ patentes concedidas.

data foi avaliado em um contexto micro. Segundo o Guidelines for preparing Patent Landascape Reports publicado pela WIPO em $2015^{6}$ as análises dos documentos de patentes podem ser divididas nos níveis macro, quando se analisa volumes maiores que 10.000 documentos; análise meso, quando se trabalha entre 1.000 e 10.000 documentos; análises micro quando se trabalha com menos de 1.000 documentos. O leitor poderá verificar que no exemplo abaixo nem todos os indicadores foram utilizados. Por se tratar de uma análise exclusivamente feita para tecnologias depositadas no Brasil, foram excluídos indicadores que se utilizam de variáveis dependentes de depósitos em outros países, como PatT, por exemplo. Outros indicadores foram omitidos apenas por conveniência e limitação de espaço. Caberá ao analista utilizar tais quais ou tanto quantos indicadores sejam necessários para que se tenha o panorama tecnológico que se deseja analisar.

\section{Perfil do patenteamento de tecnologias relacionadas a terras raras no Brasil}

No dia 07 de abril de 2020 foi feita uma busca no site $w w w$. lens.org utilizando as palavras chave "terras raras" em all fields e limitando a busca por jurisdiction "Brasil". Foram recuperados
439 depósitos de patentes, representando 374 famílias de patentes. Conforme explicitado anteriormente, apenas as famílias de patentes foram analisadas, uma vez que o grande grupo de patentes inclui repetições das tecnologias que foram depositadas em outros países. Como a análise possui o foco específico em depósitos feitos no Brasil, não faria sentido analisar o grupo de 439 documentos para verificar panoramas tecnológicos fora do país.

A Figura 2 se refere à construção da série histórica de depósitos no Brasil. Nota-se que não há nenhuma tendência que possa ser, em um primeiro momento, facilmente identificada. A transdisciplinaridade pode ser verificada neste momento, uma vez que aspectos técnicos, históricos, econômicos e até mesmo políticos, deveriam ser cruzados com os dados obtidos na análise da série histórica para entender melhor a razão dos picos e vales ilustrados na Figura 2.

De forma a tentar prever a saturação tecnológica do que está apresentado nas patentes de terras raras no Brasil, o gráfico da Figura 3 foi construído utilizando o número acumulado de depósitos de patentes por ano. A partir da análise desse gráfico, diversos ajustes matemáticos podem ser feitos para se tentar prever o "futuro" do desenvolvimento nessa área. No livro de Porter et al., ${ }^{22}$ publicado em 2011, vários desses ajustes matemáticos, bem como equações,

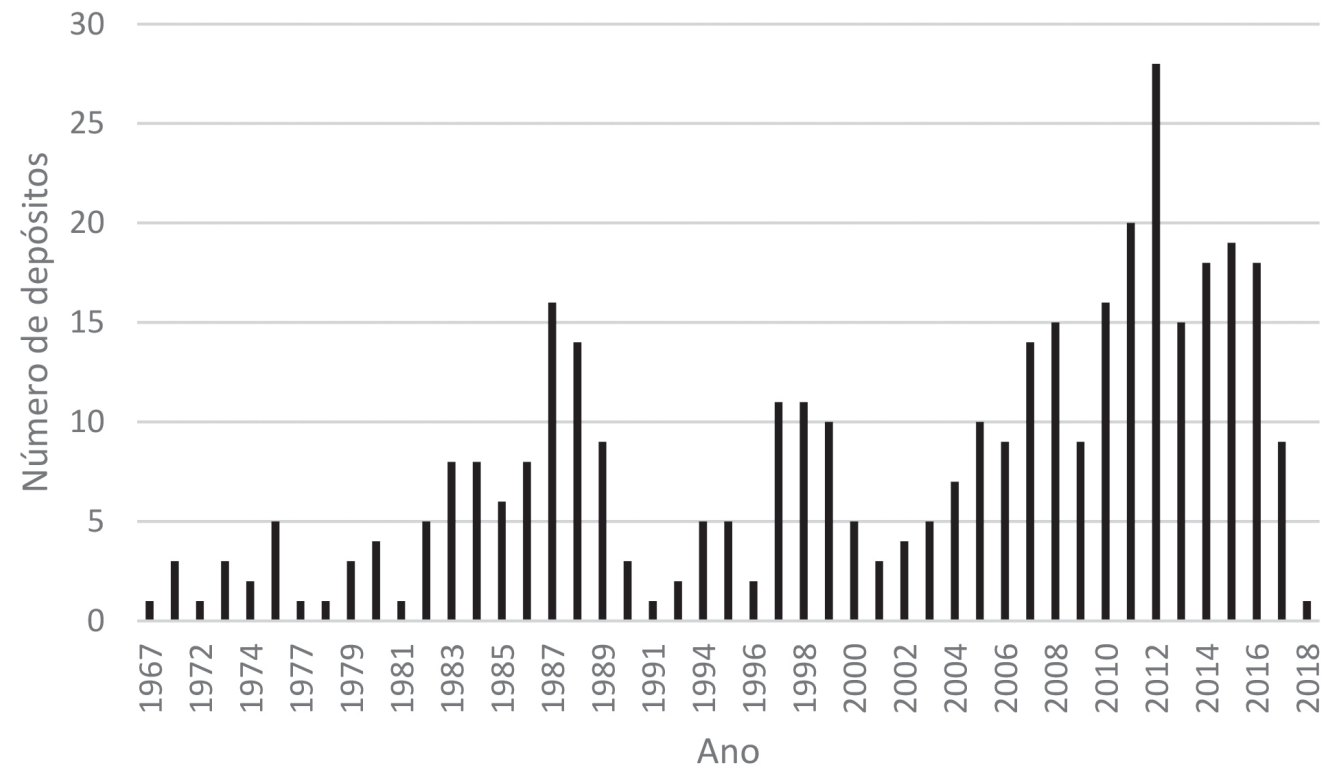

Figura 2. Série temporal dos depósitos de patentes sobre terras raras no Brasil 
vantagens e desvantagens de se utilizar um ou outro método poderá ser encontrado.

Para simplificar a análise, a título ilustrativo das possibilidades, foi feito um ajuste matemático da distribuição dos pontos apresentados na Figura 3 utilizando a distribuição de Boltzmann. A partir disso, um novo gráfico (Figura 4) foi obtido (c.f. Equação 21).

Pela análise da curva apresentada na Figura 4, tem-se uma "previsão" de desenvolvimento tecnológico das terras raras até o ano de 2300. No entanto, considerando-se a quantidade anual de depósitos no Brasil, as tecnologias em terras raras podem ser definidas como emergentes até o ano de 2100, quando teriam um rápido crescimento atingindo a saturação tecnológica em 2200. Tal saturação tende a ocorrer caso a frequência de depósitos deste tipo de tecnologia no país ocorra conforme as últimas cinco décadas. É preciso frisar que isso é uma análise matemática simplificada que não leva em consideração fatores externos que, provavelmente, deslocariam essa curva para mais perto ou longe da atualidade. Além disso, analisa apenas a frequência de depósitos anuais. Para se ter uma análise mais fidedigna sobre a saturação de tecnologias em terras raras, de um modo geral, esse tipo de análise deveria ser feito num contexto mundial, uma vez que os desenvolvedores de tecnologias nessa área, em sua grande maioria, não são originários do Brasil.

$$
y=A_{2}+\frac{A_{1}-A_{2}}{1+e^{\frac{x-x_{0}}{d x}}} ; \mathrm{R}^{2}=0,989
$$

em que as variáveis da Equação 21 são: $\mathrm{A}_{1}=-50,913 \pm 29,912$; $\mathrm{A}_{2}=179420,309 \pm 2,810 \cdot 10^{7} ; \mathrm{x}_{0}=2156,897 \pm 3670,400$; $\mathrm{dx}=23,084 \pm 8,139$.

Como pode ser visto na Figura 5, os grandes depositantes de patentes no Brasil são basicamente empresas estrangeiras, com a presença de algumas poucas universidades. Um total de 220 depositantes distintos foram encontrados na análise patentométrica e, para melhor, visualização desses dados, os 34 mais relevantes foram considerados para a construção do gráfico. Uma atenção especial deve ser dada para o único representante brasileiro no gráfico: a empresa Vale S.A. que ocupa a $33^{\mathrm{a}}$ posição.

Considerando o grupo de famílias de tecnologias depositadas

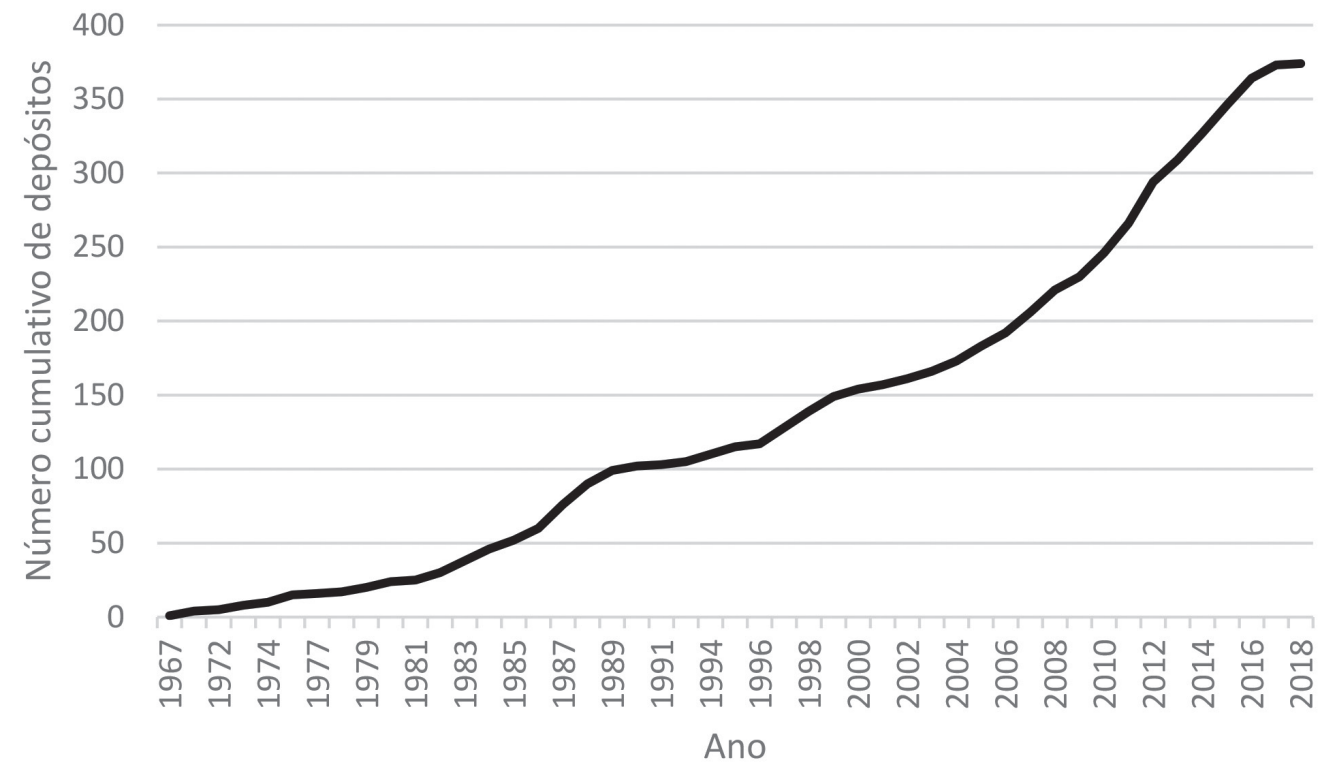

Figura 3. Construção da curva S para tecnologias em terras raras no Brasil

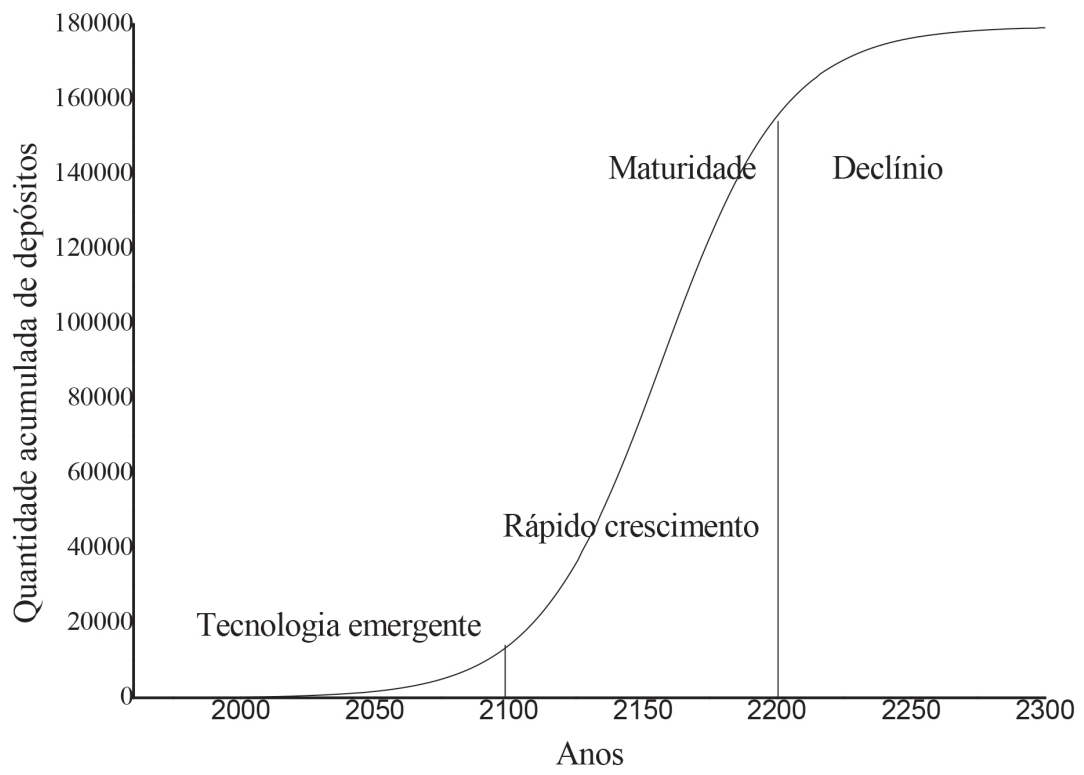

Figura 4. Ajuste matemático da curva S de proteção de tecnologias em terras raras no Brasil 


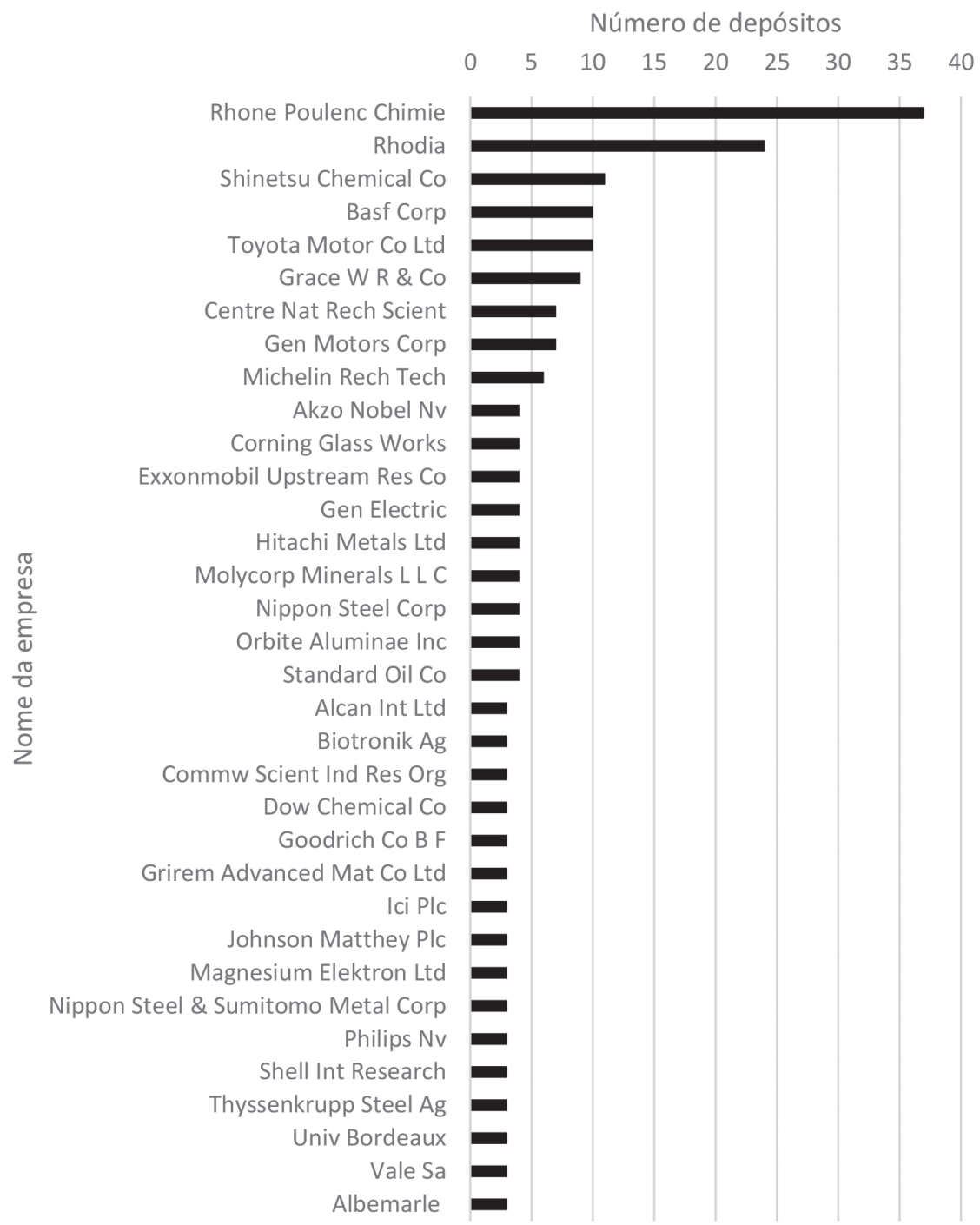

Figura 5. Empresas com pelo menos dois depósitos de patentes sobre terras raras no Brasil num total de 220 depositantes distintos

no Brasil, optou-se pela análise do maior nível de detalhamento dos códigos internacionais de patentes que se referem tecnicamente ao conteúdo do documento depositado. Sendo assim, com esses dados foi construído o gráfico mostrado na Figura 6. Os significados das subclasses de IPC analisados podem ser encontrados em detalhes no site ipc.inpi.gov.br.

De forma resumida, apresentamos abaixo o significado dos IPC identificados na Figura 6, são eles:

- C01F17/00 - compostos de metais de terras raras;

- C22B59/00 - obtenção de metais de terras raras;

- C22C38/00 - ligas ferrosas, ex. ligas de aço;

- B01J23/10 - catalisadores compreendendo metais ou óxidos ou hidróxidos de metal;

- H01F1/057 - materiais magnéticos e elementos IIIA, ex. $\mathrm{Nd}_{2} \mathrm{Fe}_{14} \mathrm{~B}$;

- C07B61/00 - purificação, separação, estabilização;

- H01F1/08 - materiais magnéticos prensados, sinterizados ou unidos;

- H01F41/02 - aparelhos ou processos especialmente adaptados para a fabricação ou montagem de ímãs, indutâncias ou transformadores; aparelhos ou processos especialmente adaptados para materiais de fabricação, caracterizados por suas propriedades magnéticas para a fabricação de núcleos, bobinas ou ímãs;

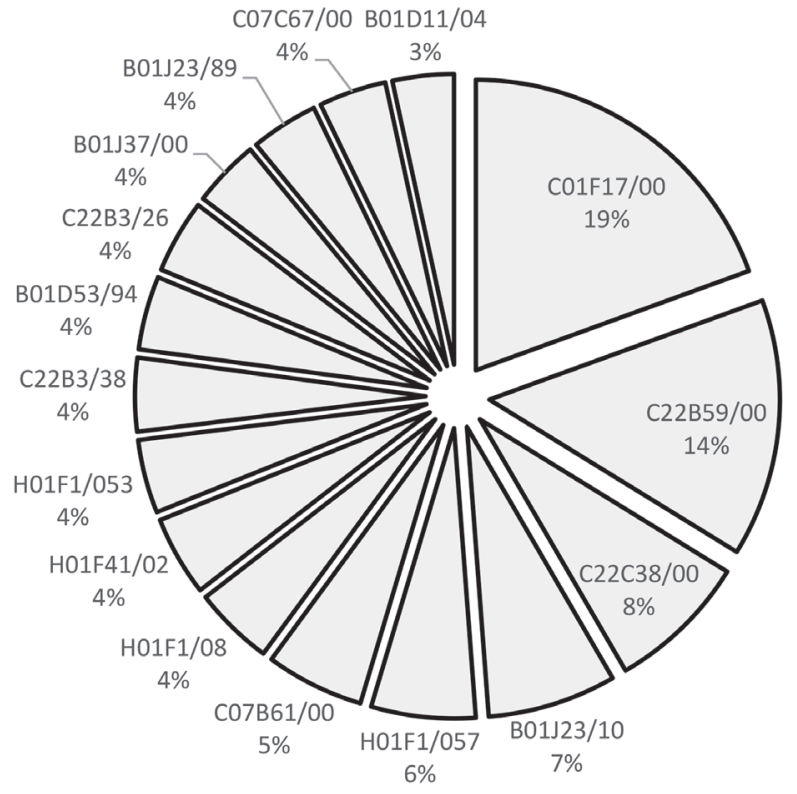

Figura 6. Concentração tecnológica das patentes depositadas no Brasil referentes às terras raras, medidas pelos quinze IPC mais relevantes de um total de 56 classes distintas e 121 subclasses 
- H01F1/053 - materiais magnéticos contendo metais de terras raras;

- C22B3/26 - extração de compostos metálicos de minérios ou concentrados por processos úmidos por extração líquido-líquido utilizando compostos orgânicos;

- C22B3/38 - descrição do C22B3/26 acrescido do elemento fósforo;

- B01D53/94 - separação de gases ou vapores; recuperação de vapores de solventes voláteis de gases; purificação química ou biológica de gases residuais, ex. gases de exaustão do motor, fumaça, gases de combustão ou aerossóis por processos catalíticos;

- C07C67/00 - preparação de ésteres de ácido carboxílico;

- B01J23/89 - catalisadores compreendendo metais ou óxidos ou hidróxidos de metal combinado com metais nobres;

- B01J37/00 - processos, em geral, para a preparação de catalisadores; processos, em geral, para ativação de catalisadores;

- B01D11/04 - extração com solventes de soluções líquidas.

Algumas bases de dados de patentes disponibilizam nos arquivos exportados os campos de título, de resumo e de reivindicações. No caso da base utilizada, o Lens disponibiliza apenas o título, porém esse já é responsável por grande parte das informações que interessam. Os títulos das patentes, normalmente, carregam consigo a categoria a ser protegida, tais como: processos e métodos de obtenção ou síntese, a composição ou o próprio produto em si e, em alguns casos, o próprio uso do produto reivindicado. Portanto, o objetivo macro da proteção poderá ser bem entendido pela análise do título, sob um contexto de big data analysis, sem que seja extremamente necessária uma outra análise aprofundada nos resumos ou reivindicações. O campo dos títulos poderá ser analisado por ferramentas de análise de repetição de palavras, ou mesmo por ferramentas de análise semântica de big data. Para este caso, foi utilizado um software disponível no site https://www.wordclouds.com/ para verificar o campo título das 374 patentes recuperadas. A recorrência dessas palavras foi reproduzida em um formato gráfico, conforme demonstrado na Figura 7.

Para a análise da Figura 7, devem ser excluídas as chamadas palavras "stop words", como elementos de ligação, por exemplo. A recorrência das palavras categorizantes dos tipos de proteção método, preparação, composição, produção, e outras, sempre serão termos de alta relevância, uma vez que aparecem em praticamente todos os tipos de patentes. Ainda dentro dessa nuvem de palavras, é

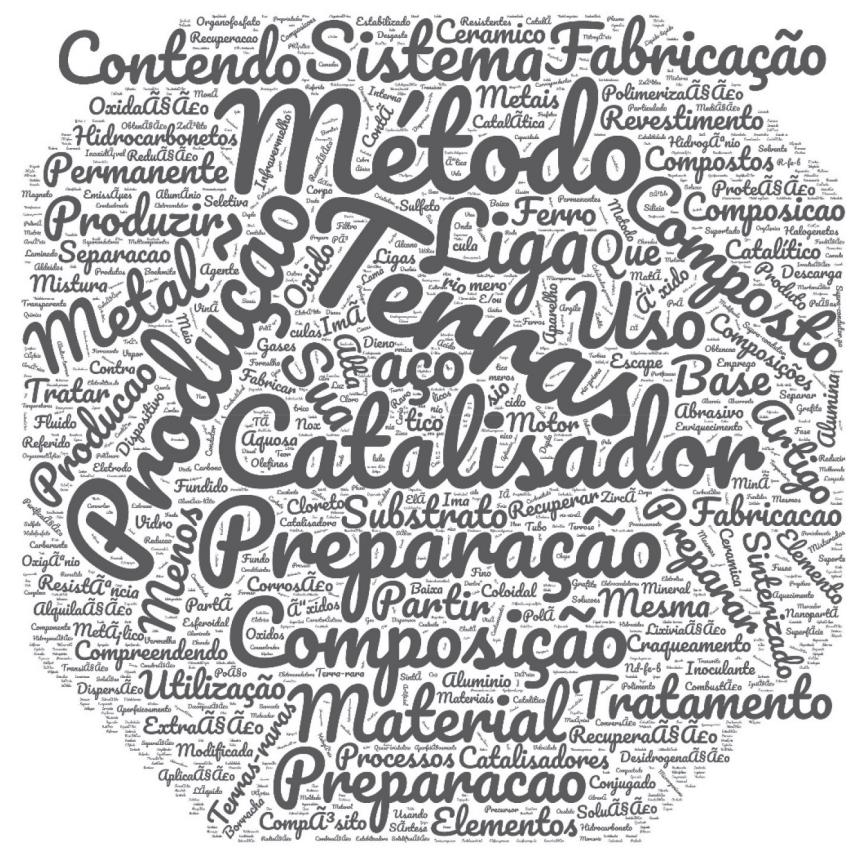

Figura 7. Nuvem de palavras utilizando o texto dos títulos das patentes

facilmente perceptível a recorrência dos termos "catalisador", "liga", "aço". Esse tipo de análise pode e deve ser cruzado com a análise dos IPC, a fim de detectar quais campos tecnológicos possuem maior relevância para o setor analisado.

A utilização de ferramentas mais sofisticadas possibilita o cruzamento dos dados para formar análises de rede social e nós, que permitem detectar possíveis gargalos e possibilidades de interação entre tecnologias. Assim, é possível obter novas e mais completas análises das tecnologias, bem como detectar possibilidades de geração de novos negócios e interações entre agentes internos e externos. A literatura já dispõe de diversos tipos de trabalho nesse contexto, e para não ser muito extensivo no presente artigo, recomenda-se a leitura cuidadosa dos trabalhos de Aaldering et al. ${ }^{25}$ e Pereira et al. ${ }^{26}$

Os cinco mais expressivos depositantes de patentes na área analisada foram avaliados segundo os indicadores propostos pelo professor Holger Ernst. Os dados estão expressos na Tabela 2 e a visualização

Tabela 2. Índices de qualidade para as cinco empresas com mais depósitos no Brasil na área de terras raras

\begin{tabular}{|c|c|c|c|c|c|}
\hline Indicador / Empresa & $\begin{array}{l}\text { Rhone Poulenc } \\
\text { Chimie }\end{array}$ & Rhodia & $\begin{array}{c}\text { Shinetsu Chemical } \\
\text { Co. }\end{array}$ & BASF Corp. & $\begin{array}{c}\text { Toyota Motor Co. } \\
\text { LTD. }\end{array}$ \\
\hline Atividade patentária & 37 & 24 & 11 & 10 & 10 \\
\hline Compartilhamento de tecnologias & 0,099 & 0,064 & 0,029 & 0,027 & 0,027 \\
\hline Ênfase em P\&D* & 0,089 & 0,022 & 0,157 & 0,001 & 0,006 \\
\hline Intensidade de cooperação & 0 & 1 & 2 & 0 & 3 \\
\hline Compartilhamento de tecnologias concedidas & 0 & 3 & 5 & 2 & 0 \\
\hline Escopo tecnológico & 177 & 148 & 35 & 39 & 23 \\
\hline Escopo internacional & 639 & 410 & 153 & 152 & 69 \\
\hline Frequência média de citações & 0 & 0 & 0 & 0 & 0 \\
\hline Qualidade média das patentes & 816 & 562 & 195 & 193 & 95 \\
\hline Força das patentes & 30192 & 13488 & 2145 & 1930 & 950 \\
\hline Compartilhamento de tecnologias ${ }^{\#}$ & 0,619 & 0,277 & 0,440 & 0,039 & 0,019 \\
\hline Compartilhamento relativo de tecnologias & 1,000 & 0,447 & 0,071 & 0,064 & 0,031 \\
\hline
\end{tabular}

*Indicador adaptado para considerar apenas as patentes depositadas no Brasil. Atividade pantentária dividida pelos números de depósitos da empresa (i) no Brasil. Depositante (número total de depósitos de patentes no Brasil): Rhone Poulenc Chimie (415), Rhodia (1075), Shinetsu Chemical Co. (70), BASF Corp. (6879), Toyota Motor Co. LTD. (1494). "Baseado na força das patentes. 


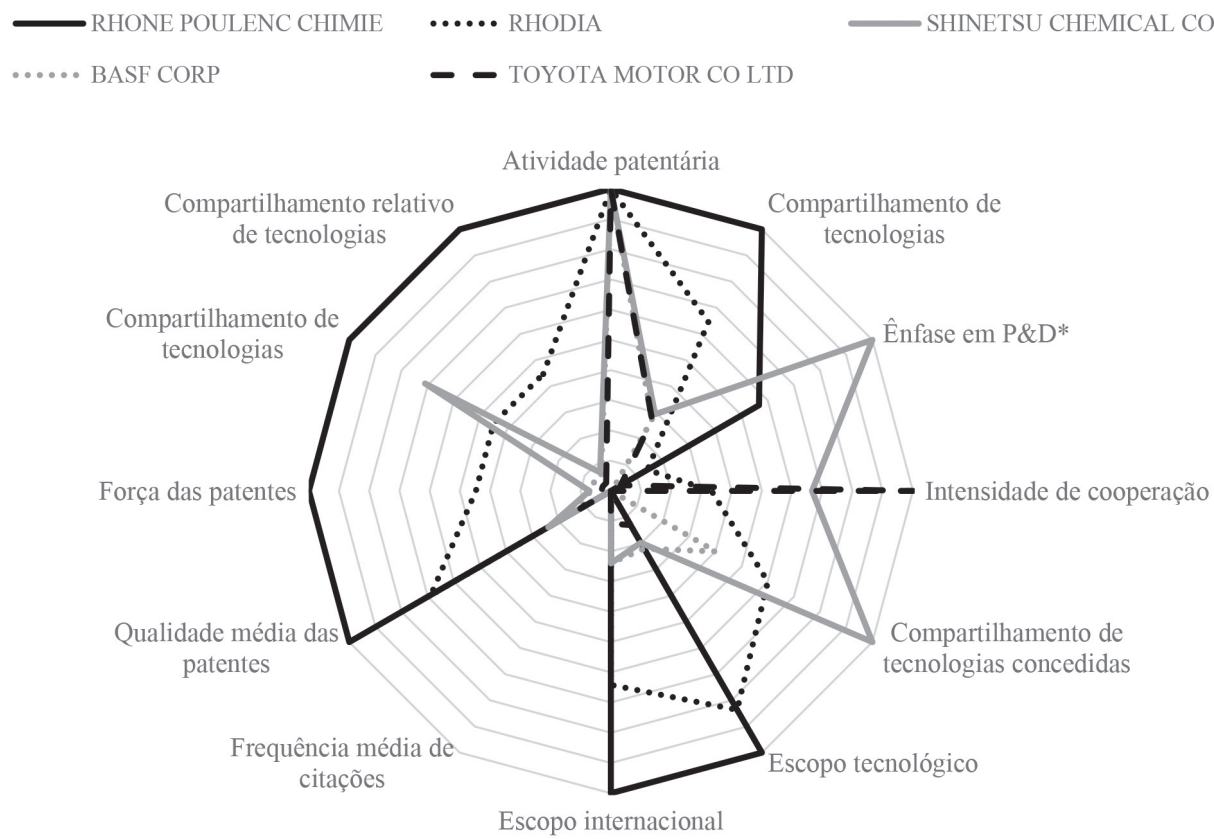

Figura 8. Gráfico radar para as empresas analisadas segundo os indicadores de Holger Ernst

gráfica pode ser conferida na Figura 8. A Figura 8 foi normalizada para melhor visualização dos eixos. Pelos índices analisados a empresa Rhone Poulenc Chimie é a que apresenta um índice médio maior de qualidade de patentes. Novamente, a transdisciplinaridade é uma ferramenta fundamental nesse tipo de análise, uma vez que podem ser incluídos aqui dados econômicos dessas empresas de forma a aumentar a qualidade do mapeamento. Dados de produção tecnológica obtidos pelos índices de patenteamento, cruzados com dados de participação de mercado, ativos intangíveis, $\mathrm{P} \& \mathrm{D}$, dentre outros, quando analisados em conjunto, podem indicar o grau de utilização do segredo industrial como estratégia de proteção dos resultados de P\&D das empresas.

Relacionando os principais IPC (Figura 6) e os mais expressivos depositantes (Tabela 2), é possível estabelecer uma matriz de relacionamento para identificar a concentração tecnológica dos atores envolvidos e comparar o foco de desenvolvimento das tecnologias que envolvem as terras raras.

Por meio da Tabela 3 é possível visualizar que, embora o campo macro tecnológico seja o mesmo, existem diferentes concentrações tecnológicas dos IPC por empresa. Verifica-se com esse tipo de análise, por exemplo, que as empresas Rhodia e Rhone Poulenc Chimic possuem foco de desenvolvimento no campo C01F17/00, enquanto a BASF possui predominância nos campos B01D53/94 e B01J23/89. A construção deste tipo de cenário possibilita uma comparação de concorrentes mais específica, além de um maior detalhamento de P\&D\&I da empresa, que pode ser complementado com dados econômicos, políticos, mercadológicos e de outras propriedades intelectuais.

Dentre as muitas outras análises possíveis utilizando a patentometria, certamente a análise técnica em nível micro para publicação de caráter mais técnico é fundamental. Mesmo que a análise em escala micro contemple uma quantidade inferior a 1000 documentos, a avaliação de todos em detalhes é uma das barreiras em sua execução. Dessa forma, a seleção de documentos prioritários para realização de uma análise mais detalhada é fator fundamental para a exequibilidade do projeto. Considerando os vários subgrupos de patentes existentes, elenca-se aqueles que podem servir de filtro para a análise: patentes com depósitos mais recentes, patentes triádicas, patentes das empresas

Tabela 3. Relação entre os principais IPC e as cinco empresas com mais depósitos no Brasil na área de terras raras

\begin{tabular}{|c|c|c|c|c|c|}
\hline IPC Grupo & BASF Corp. & Rhodia & Rhone Poulenc Chimie & Shinetsu Chemical Co. & Toyota Motor Co. LTD. \\
\hline $\mathrm{C} 01 \mathrm{~F} 17 / 00$ & 1 & 9 & 28 & 1 & \\
\hline $\mathrm{C} 22 \mathrm{~B} 59 / 00$ & & 1 & 13 & 2 & \\
\hline B01J23/10 & 1 & 9 & 3 & & \\
\hline H01F1/057 & & & & 4 & 4 \\
\hline H01F41/02 & & & & 4 & 4 \\
\hline H01F1/08 & & & & 4 & 3 \\
\hline H01F1/053 & & & & 5 & 2 \\
\hline B01D53/94 & 2 & 3 & 1 & & 1 \\
\hline $\mathrm{C} 22 \mathrm{~B} 3 / 38$ & & & 6 & & \\
\hline $\mathrm{C} 22 \mathrm{~B} 3 / 26$ & & & 4 & 2 & \\
\hline B01J37/00 & 1 & & 1 & & \\
\hline B01J23/89 & 2 & & & & \\
\hline Total Geral & 7 & 22 & 56 & 22 & 14 \\
\hline
\end{tabular}


mais expressivas, patentes com maiores índices de citação, patentes de determinados IPC em específico. Qualquer um desses outros delimitadores irá auxiliar o analista a escolher o subgrupo de análise, desde que justificada essa escolha.

Em caráter ilustrativo, as patentes depositadas pela empresa Rhone Poulenc Chimie, que pelos índices da Tabela 2 apresentaram uma maior qualidade, têm seus títulos reproduzidos abaixo (Tabela 4). Embora as patentes da empresa Rhone Poulenc Chimie sejam relativamente antigas, essas podem servir de base para as tecnologias mais modernas. Os aspectos de cada tecnologia relatada não serão detalhados por fugir do objetivo desse artigo, que pretende apresentar algumas das várias possibilidades da patentometria em si e não o detalhamento de uma área tecnológica específica.

Documentos complementares ajudarão em uma interpretação mais assertiva do panorama das tecnologias em terras raras depositadas no Brasil. São eles: o artigo publicado na revista in the mine (2018), de Mathias Heider; ${ }^{27}$ o artigo publicado na revista Química Nova (2014), de Paulo C. de Sousa Filho e Oswaldo A. Serra, ${ }^{28}$ e o

Tabela 4. Patentes depositadas no Brasil pela empresa Rhone Poulenc Chimie

\begin{tabular}{ll}
\hline $\mathrm{N}^{\mathrm{o}}$ Publicação & Título da Patente \\
\hline
\end{tabular}

BR 8902293 A Abrasivo

BR 8403754 A Composição de polimento a base de cério e processo para sua fabricação

BR 8402229 A Composição de polimento e processo para a sua fabricação

BR 8905084 A Dispersões de halogenetos de terras raras em meio aquoso

BR 8905339 A Dispersões de halogenetos de terras raras, em meio oleoso, processo de obtenção das mesmas

BR 9002001 A Halogenetos de terras raras desidratados e processo de desidratação dos mesmos

BR 9002961 A Misturas desidratadas de halogenetos de terras raras e de metais alcalino terrosos ou alcalinos, e processo de sua produção

BR 8906507 A Óxido de terra rara e processo para sua preparação

BR 8700668 A Processo de eliminação de chumbo de terras raras

BR 8901082 A Processo de fabricação de materiais supercondutores e pó cerâmico assim obtido

BR 9101788 A Processo de fabricação de um oxalato duplo de terras raras e de amônio, oxalato duplo de amônio e de terra rara e oxido de terras raras

BR 9104904 A Processo de fabricação de um oxalato duplo de terras raras e de amônio, processo de fabricação de oxido de terras raras e oxido de terras raras;

BR 8802950 A Processo de obtenção de um oxido de terra rara e oxido de terra rara

BR 9502568 A Processo de preparação de um composto dispersível à base de pelo menos uma terra rara composto à base de pelo menos uma terra rara e de um óxido de terra rara e eventualmente de pelo menos um outro elemento suspensão coloidal composição catalítica processos de preparação das mesmas utilização e catalisador

BR 9601067 A Processo de preparação de um sulfeto de terra rara

BR 8901865 A Processo de preparação de boretos de terras raras

BR 8806171 A Processo de preparação de boretos de terras raras

BR 8804802 A Processo de preparação de um pó fino supracondutor, pó cerâmico e material cerâmico sinterizado supracondutor

BR 8804803 A Processo de preparação de um pó fino supracondutor, pó cerâmico e material cerâmico sinterizado supracondutor

BR 8804801 A Processo de preparação de um pó fino supracondutor, pó cerâmico e material cerâmico sinterizado supracondutor

BR 8804714 A Processo de preparação de uma dispersão coloidal de um composto de terra rara e dispersão coloidal

BR 8600098 A Processo de purificação de soluções aquosas de sais de terras raras

BR 9004677 A Processo de recuperação dos valores de terras raras contidos no gipso

BR 8501508 A Processo de separação das terras raras por extração líquido líquido

BR 8801283 A Processo de separação de terras raras contidas em uma solução aquosa

BR 8806226 A Processo de separação de tório e de terras raras de um concentrado de fluoretos de pelo menos um destes elementos

BR 8601305 A Processo de separação do cério e das terras raras a partir de uma fase aquosa inicial

BR 8701213 A Processo de separação do neodímio ou do didimio das terras raras contidas na bastnaesita

BR 8206321 A Processo de separação e recuperação do uranio, do tório, das terras raras e eventualmente do ferro por extração líquido-líquido

BR 9100372 A Processo de síntese de compostos binários de enxofre, composto sulfurado de terras raras, mistura de compostos sulfurados, sesquissulfetos de terras raras e sulfeto de metal de transição

BR 8004114 A Processo de tratamento de mistura de óxidos de terras paras e de gálio

BR 9408456 A Processo de tratamento de sujidades fuliginosas adjuvante para carburante de motor de combustão interna e fuligem à base de terra rara trivalente

BR 9000987 A Processo de tratamento de um mineral de terras raras

BR 8604289 A Processo de tratamento de um mineral de terras raras

BR 9004544 A Processo de tratamento do mineral

BR 8008474 A Processo para fabricação de composições de polímero

BR 7901424 A Processo para separação de terras raras 
trabalho publicado pelo Centro de Gestão em Estudos Estratégicos do MCTIC (2013). ${ }^{29}$

\section{COMENTÁRIOS FINAIS}

O mapeamento de tecnologias utilizando a patentometria, embora seja uma área pouquíssima explorada no Brasil, é uma ferramenta fundamental para os tomadores de decisões estratégicas, quando nos referimos à inovação tecnológica. A prospecção tecnológica tradicional feita documento a documento permite uma análise extremamente detalhada do conteúdo tecnológico descrito em patentes, entretanto, não permite a visão do todo de forma estratégica. A análise de big data, em qualquer que seja a área, possibilita uma visão ampla sobre tendências e concentrações de informação estratégica que podem ser usadas para definir prioridades de ação. Além disso, a patentometria, considerando a análise simultânea de várias variáveis, pode mostrar resultados nem um pouco intuitivos quando comparada a análise monodimensional.

A patentometria é uma área altamente transdisciplinar, assim, a análise bem sucedida dos resultados irá requerer profissionais não somente das áreas técnicas (ciências duras ou engenharias), mas também da economia, tecnologia da informação, direito, administração, engenharia de produção e outras, conforme os objetivos pretendidos. No presente artigo, de forma ilustrativa, foi apresentado uma das várias possibilidades de se fazer o mapeamento tecnológico, utilizando como exemplo tecnologias patenteadas e relacionadas às terras raras no Brasil. A intepretação do mapa obtido por profissionais da área das engenharias ou química certamente tenderá a ser mais técnica, considerando o avanço técnico ao longo do tempo das tecnologias apresentadas. $\mathrm{O}$ mesmo mapa ao ser analisado por profissionais da administração ou economia pode ter uma interpretação voltada para uma análise das indústrias envolvidas nas pesquisas do setor tecnológico em questão, o efeito dos ativos de patentes na liderança em inovação dessas indústrias, a implicação dessas patentes nos indicadores internacionais de desenvolvimento e inovação, a relação entre a participação no mercado de determinadas empresas, posição econômica no cenário global, relacionamento com inovação e a relação entre depósitos de patentes e inovação para essas empresas. Dentre todos os tipos de análises possíveis, certamente uma das mais valiosas seja a previsão das tendências futuras, que permite identificar o surgimento de tecnologias inovadoras e possíveis mercados a serem criados. Além disso, a análise de possíveis conexões tecnológicas para o licenciamento cruzado e previsões de substituições de tecnologias em uso por outras mais atrativas, também possuem destaque dentro de uma gama de opções.

Por fim, independentemente de quais sejam as intenções do analista, os conhecimentos básicos de propriedade intelectual são fundamentais quando se trabalha com patentometria. O que apresentamos aqui são apenas algumas das possibilidades de uso dessa ferramenta. Nesse sentido, o analista poderá, certamente, com criatividade e respeitando os fundamentos da propriedade intelectual, aprofundar o detalhamento da análise de seu mapa de tecnologias e obter os mais diversos cenários que auxiliarão os tomadores de decisão, gestores de portfólios de tecnologia e inovação. Como consequência, a análise dos cenários poderá contribuir para minimizar os riscos de investimento de recursos financeiros e tempo em áreas tecnológicas estratégicas ou portadoras de futuro.

\section{AGRADECIMENTOS}

Os autores agradecem o programa de pós graduação profissional em inovação tecnológica e propriedade intelectual da UFMG e ao CNPq pela bolsa concedida.

\section{REFERÊNCIAS}

1. Tanaka, Y.; Inui, T. Em Portland International Conference on Management of Engineering and Technology (PICMET), Portland, USA, 2016.

2. Anônimo; Patent Statistics Manual, OECD Publishing: Paris, 2009, p. 158.

3. Ernst, H.; World Pat. Inf. 2003, 25, 233.

4. Altuntas, S.; Dereli, T.; Kusiak, A.; Technological Forecasting and Social Change 2015, 96, 202.

5. Ernst, H.; Patentinformationen für die strategische Planung von Forschung und Entwicklung, Springer: Wiesbaden, 2013.

6. Trippe, A.; Guidelines for Preparing Patent Landscape Reports, WIPO: Switzerland, 2015.

7. Kupfer, D.; Tigre, P. Em Modelo SENAI de prospecção: documento metodológico. Papeles de la Oficina Técnica, Caruso, L. A.; Togre, P. B., eds.; OIT/CINTERFOR: Montevideo, 2004, vol. 14.

8. Teixeira, L. P.; Prospecção Tecnológica: importância, métodos e experiências da Embrapa Cerrados, Embrapa: Distrito Federal, 2013.

9. Coelho, G. M.; Prospecção tecnológica: metodologias e experiências nacionais e internacionais: tendências tecnológicas: nota técnica 14, Turbulência Consultoria Técnica Ltda. Estratégias de Comunicação: Rio de Janeiro, 2003.

10. Dernis, H.; Guellec, D.; Science Technology Industry Review 2002, 27, 129.

11. Speziali, M. G.; Sinisterra, R. D.; Quim. Nova 2015, 38, 1132.

12. Mattos, L. H. S.; Speziali, M. G.; World Pat. Inf. 2017, 51, 57.

13. Faria, L. R.; Cartaxo, R. J. Á.; Leão, V. A.; Speziali, M. G.; Hydrometallurgy 2018, 175, 155.

14. Abbas, A.; Zhang, L.; Khan, S. U.; World Pat. Inf. 2014, 37, 3.

15. Lo, Y.-C.; Rensi, S. E.; Torng, W.; Altman, R. B.; Drug Discovery Today 2018, 23, 1538.

16. Mahjour, B.; Shen, Y.; Liu, W.; Cernak, T.; Nature 2020, 580, 71.

17. Sandfort, F.; Strieth-Kalthoff, F.; Kühnemund, M.; Beecks, C.; Glorius, F.; Chem 2020, 6, 1379, doi: 10.1016/j.chempr.2020.02.017.

18. Howarth, A.; Ermanis, K.; Goodman, J. M.; Chem. Sci. 2020, 11, 4351.

19. www.wipo.int/standards/en/pdf/03-09-01.pdf, acessada em agosto 2020.

20. https://data.oecd.org/rd/triadic-patent-families.htm, acessada em agosto 2020.

21. Sternitzke, C.; Scientometrics 2009, 81, 91.

22. Porter, A. L.; Cunningham, S. W.; Banks, J.; Roper, A. T.; Mason, T. W.; Rossini, F. A.; Forecasting and Management of Technology, Wiley: Hoboken, 2011.

23. Soete, L. G.; Wyatt, S. M. E.; Scientometrics 1983, 5, 31.

24. Balassa, B.; The Manchester School 1961, 33, 99.

25. Aaldering, L.; Leker, J.; Song, C. H.; J. Cleaner Prod. 2019, 212, 362.

26. Pereira, C. G.; Picanco-Castro, V.; Covas, D. T.; Porto, G. S.; Nat. Biotechnol. 2018, 36, 585.

27. www.inthemine.com.br/site/a-evolucao-das-terras-raras-no-brasil/, acessada em agosto 2020.

28. Sousa Filho, P. C.; Serra, O. A.; Quim. Nova 2014, 37, 753.

29. Anônimo; Usos e aplicações de Terras Raras no Brasil: 2012-2030, Centro de Gestão e Estudos Estratégicos: Brasília, Brasil, 2013. 